\title{
O tempo de balanço como variável preditiva da doença de Parkinson
}

\author{
Swing time as a predictive variable for Parkinson's disease \\ Tiempo de equilibrio como variable predictora de la enfermedad de Parkinson \\ Lucas Resende Sousa', Bárbara Crystian Rodrigues Martins², Lucyana Teodoro de Oliveira ${ }^{3}$, \\ Camilla Zamfolini Hallal ${ }^{4}$
}

RESUMO | Atualmente, a doença de Parkinson (DP) tem seu diagnóstico baseado apenas na observação clínica de uma combinação de sintomas, o que pode levar ao diagnóstico tardio, uma vez que alguns indivíduos podem ter a doença por 5 a 10 anos antes de serem diagnosticados. O objetivo do estudo foi identificar variáveis cinemáticas temporais da marcha capazes de discriminar idosos com e sem DP. 40 indivíduos foram divididos em dois grupos: grupo de idosos sem DP $(n=21)$ e com DP $(n=19)$. Dez ciclos de marcha consecutivos foram obtidos durante a marcha em velocidade de preferência, e utilizados para a análise dos dados. Realizou-se uma análise discriminativa para determinar um modelo preditor de alterações na marcha característico da DP e calculado com base na especificidade e sensibilidade de cada variável analisada, utilizando-se variáveis cinemáticas temporais. A variável com valor discriminativo de sensibilidade e especificidade foi o tempo de balanço, o que pode classificá-la como a variável com grande potencial preditivo da presença ou não da DP; o ponto de corte encontrado para essa variável foi de 0,48segundos. A análise cinemática da marcha permite discriminar um grupo de indivíduos com DP de um grupo de indivíduos saudáveis com alta sensibilidade e especificidade, por meio do tempo de balanço, menor no grupo acometido pela doença (corte de 0,48segundos).

Descritores | Doença de Parkinson; Marcha; Cinemática; Diagnóstico Precoce.

ABSTRACT I Currently, Parkinson's Disease (PD) is diagnosed based only on the clinical observation of a symptom combination, which can lead to late diagnosis, since some individuals have the disease for 5 to 10 years before diagnosis. The aim of this study was to identify temporal kinematic variables of gait, capable of discriminating older adults with or without PD. Forty individuals were divided into two groups: older adults without PD ( $n=21)$ and with PD ( $n=19)$. Ten consecutive gait cycles were obtained during gait at a preferred speed and then used in data analysis. Discriminative analysis was performed to determine a predictor model of gait changes, characteristic of PD, estimated based on the specificity and sensitivity of each analyzed variable, with temporal kinematic variables. The variable with discriminative value of sensitivity and specificity was swing time, which can be classified as the variable with most predictive potential of PD, and the cut-off point found for this variable was 0.48 seconds. Kinematic gait analysis allows discriminating a group of individuals with PD from a group of healthy individuals, with high sensitivity and specificity, through the swing time, which is lower in the group affected by the disease (cut-off=0.48 seconds).

Keywords | Parkinson's Disease; Gait; Kinematics; Early Diagnosis.

RESUMEN | Actualmente, el diagnóstico de la enfermedad de Parkinson (EP) se obtiene desde la observación clínica de una combinación de síntomas, lo que puede llevar a un diagnóstico tardío, ya que algunas personas pueden haber adquirido la enfermedad entre 5 y 10 años antes de la realización del diagnóstico. El objetivo del estudio fue identificar variables cinemáticas temporales de la marcha

'Universidade Federal de Uberlândia (UFU) - Uberlândia (MG), Brasil. E-mail: lucas.resende.sousa@hotmail.com. Orcid: 0000-0002-0178-0534

2Universidade Federal de Uberlândia (UFU) - Uberlândia (MG), Brasil. E-mail: barbaramfisio@gmail.com. Orcid: 0000-0003-4434-5740.

${ }^{3}$ Programa de Pós-Graduação em Ciências da Saúde, Faculdade de Medicina, Universidade Federal de Uberlândia - Uberlândia (MG), Brasil. E-mail: lucyanat.oliveira@hotmail.com. Orcid: 0000-0001-5168-1334

${ }^{4}$ Universidade Federal de Uberlândia (UFU) - Uberlândia (MG), Brasil. E-mail: camillazhallal@ufu.br. Orcid: 0000-0001-7414-7679

Endereço para correspondência: Lucas Resende Sousa - Rua Benjamin Constant, 1286, Nossa Senhora Aparecida - Uberlândia (MG), Brasil - CEP: $38400-678$ - 
capaces de diferenciar a ancianos con EP y ancianos sin EP. Se dividieron los 40 participantes en dos grupos: ancianos $\sin E P(n=21)$ y ancianos con EP ( $n=19)$. Para el análisis de datos, se obtuvieron diez ciclos de marcha consecutivos durante la marcha a la velocidad preferida. Se realizó un análisis discriminante para determinar un modelo predictivo de cambios en la marcha característicos de EP que se calcula en base a la especificidad y sensibilidad de cada variable analizada utilizando variables cinemáticas temporales. La variable con valor discriminante de sensibilidad y especificidad fue el tiempo de equilibrio, que se puede clasificar como la variable con mayor potencial para predecir la presencia o no de EP; el punto de cohorte encontrado para esta variable fue de 0,48 segundos. El análisis cinemático de la marcha tiene una alta sensibilidad y especificidad en la identificación de individuos con EP comparados a individuos sanos por medio del tiempo de equilibrio, que es menor en el grupo afectado por la enfermedad (cohorte de 0,48 segundos). Palabras clave | Enfermedad de Parkinson; Marcha; Cinemática; Diagnóstico Precoz.

\section{INTRODUÇÃO}

A doença de Parkinson (DP) foi descrita pela primeira por James Parkinson em 1817 e se caracteriza contemporaneamente pela presença de sinais cardinais, como o tremor de repouso, a bradicinesia, a rigidez e a instabilidade postural ${ }^{1}$. Além dos comprometimentos motores característicos da DP, uma considerável quantidade de pacientes apresenta algum tipo de deficiência cognitiva ${ }^{2}$.

As manifestações clínicas são ocasionadas por uma expressiva redução do neurotransmissor dopamina nos núcleos da base, devido à degeneração dos neurônios dopaminérgicos da substância negra do mesencéfalo por conta do acúmulo da proteína alfa-sinucleína nos corpos celulares desses neurônios na forma de agregados filamentosos intracelulares (corpos de Lewy) ${ }^{3,4}$.

Nesse contexto, o envelhecimento do indivíduo está fortemente ligado ao desenvolvimento da DP, em razão da aceleração da perda dos neurônios produtores de dopamina com o decorrer do tempo, o que afeta aproximadamente $2 \%$ das pessoas com 65 anos de idade 5 . A DP é considerada a segunda doença neurodegenerativa mais frequente em idosos, e estima-se que mais de 6,3 milhões de pessoas em todo o mundo tenham a doença ${ }^{6}$; nos Estados Unidos, a estimativa é de que mais de um milhão de pessoas sejam diagnosticadas com DP até $2030^{7}$.

Dentre os comprometimentos que a doença causa, as alterações na marcha são uma das que mais limitam a funcionalidade ${ }^{8}$. A dificuldade da regulação espaçotemporal, o reduzido comprimento de passada (CP), a maior frequência de passada (FP), o maior tempo do duplo apoio dos pés no chão e a maior variabilidade dos parâmetros espaço-temporais são fatores que interferem na funcionalidade na $\mathrm{DP}^{9}$. A variabilidade dos parâmetros espaço-temporais tem uma relação inversa com a estabilidade dinâmica da marcha. Nos indivíduos acometidos pela DP, há uma alteração no centro de massa causado pela instabilidade postural, muitas vezes evidente muitas em situações como mudanças de direção e velocidade ${ }^{10}$. Essas alterações levam os pacientes com DP a um maior gasto energético se comparado com as situações de marcha de indivíduos jovens e saudáveis, o que os predispõem a quedas e desfechos graves, como fraturas e óbitos ${ }^{11}$.

Atualmente, o diagnóstico da DP é baseado na observação dos sintomas. Contudo, a DP é comumente diagnosticada tardiamente; alguns indivíduos podem ter a doença de cinco a dez anos antes de serem diagnosticados ${ }^{12,13}$ e, no momento do diagnóstico, até $70 \%$ dos neurônios dopaminérgicos da substância negra podem ter sido perdidos ${ }^{14,15}$.

Haja vista o diagnóstico geralmente tardio, concomitantemente às importantes alterações da marcha que acompanham a progressão da doença, é de grande importância identificar variáveis biomecânicas da marcha que sejam capazes de discriminar idosos com e sem DP. Desse modo, é possível implementar precocemente estratégias de reabilitação física e prevenção de quedas, garantindo maior segurança, qualidade de vida e independência aos pacientes com DP.

O objetivo deste estudo foi identificar variáveis cinemáticas temporais da marcha capazes de discriminar idosos com e sem DP. Nossa hipótese é de que, em função das notórias alterações da marcha presentes de maneira progressiva na DP, existam variáveis cinemáticas temporais capazes de discriminar idosos com e sem DP com alta sensibilidade ou especificidade e identificar qual variável seria mais preditiva. 


\section{METODOLOGIA}

\section{Seleção da amostra}

Participaram do estudo 40 indivíduos divididos em dois grupos: grupo de idosos sem doença de Parkinson $(n=21)$ e grupo de idosos com doença de Parkinson ( $n=19)$. A amostra foi de conveniência e determinada de acordo com o número de participantes do projeto de extensão universitária desenvolvido em prol de pacientes com doença de Parkinson. Os indivíduos sem a doença eram participantes de um programa de atividades físicas para idosos.
O estudo respeitou o sigilo da identidade dos sujeitos de pesquisa, bem como as garantias previstas no Termo de Consentimento Livre e Esclarecido, assinado por todos os participantes.

Os critérios de elegibilidade comuns aos grupos foram: idade entre 60 e 80 anos; ausência de dor, fratura ou lesão grave em tecidos moles nos seis meses pregressos ao estudo; bem como histórico de alterações cardiovasculares, respiratórias (informações referidas pelos participantes) ou cognitivas, sendo necessário um escore acima de 24 na aplicação do mini exame do estado mental (MEEM). A Tabela 1 demonstra a caracterização da amostra.

Tabela 1. Caracterização da amostra, descrita pela média e desvio-padrão

\begin{tabular}{lcrc}
\multicolumn{1}{c}{ Características } & sem Parkinson $(\mathrm{n}=21)$ & $\begin{array}{c}\text { com Parkinson } \\
(\mathrm{n}=19)\end{array}$ & $\boldsymbol{P}$ \\
Idade (anos) & $69 \pm 2$ & $69 \pm 2$ & 0,942 \\
Homens/mulheres (n) & $10 / 11$ & $9 / 10$ & - \\
H\&Y I H\&Y II (n) & - & $9 / 10$ & - \\
Peso (kg) & $71 \pm 3$ & $73 \pm 3$ & 0,648 \\
Altura (cm) & $161 \pm 2$ & $160 \pm 2$ & 0,877
\end{tabular}

H\&Y: Escala Hoehn Yahr de classificação e progressão da Doença de Parkinson.

Em adição, os participantes sem DP se enquadraram nos seguintes critérios: ausência de histórico de doenças neurológicas e praticantes de atividades físicas por no mínimo seis meses pregressos ao estudo, pelo menos três vezes por semana. Para o grupo de idosos com DP os critérios foram: diagnóstico de doença de Parkinson idiopática classificados nos estágios de I a II da escala de Hoehn Yahr (HY $)^{16}$. Pacientes nesses estágios iniciais de classificação da doença ainda são ativos funcionalmente e realizam marcha independente, ou seja, indivíduos sem comprometimentos tardios, o que permite uma identificação precoce.

Os sujeitos de pesquisa deveriam realizar fisioterapia por no mínimo seis meses pregressos ao estudo, pelo menos três vezes por semana. A atividade de fisioterapia era controlada, com atenção voltada para treinamento de equilíbrio, marcha em diversas situações, alongamentos e fortalecimento muscular. Além disso, não poderiam estar em fase de adaptação farmacológica e todos os procedimentos de coleta foram realizados na fase on dos medicamentos para DP.

\section{Instrumentos}

Para a coleta de dados cinemáticos, utilizou-se o sistema de sensores de contato foot switch $\left(\right.$ Noraxon $\left.^{\circledR}\right)$, colocado no calcâneo e na base do hálux de forma bilateral dos participantes. A Figura 1 mostra a localização dos sensores.

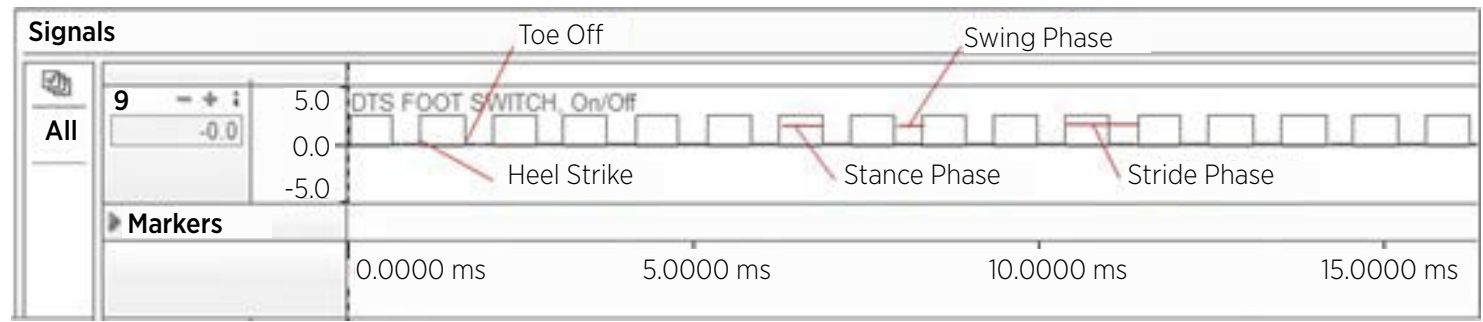

Figura 1. Sinal dos sensores de pressão usados para a determinação do início e final da fase de apoio, fase de balanço, passo e passada 


\section{Procedimentos para coleta de dados}

Antes dos procedimentos de avaliação da marcha, os voluntários foram instruídos acerca de todos os procedimentos de avaliação e familiarizados com o ambiente de coleta, os equipamentos e a tarefa no mesmo dia da coleta.

Os voluntários foram orientados, por meio de estímulo verbal, a andar sobre a passarela na velocidade que caminham rotineiramente. A atividade de marcha nesta condição foi realizada por três vezes consecutivas, e a média das tentativas foi utilizada para a análise dos dados.

A avaliação da marcha foi realizada em um tapete de dez metros de comprimento por dois metros de largura. Os primeiros dois metros e os últimos dois metros de comprimento da passarela foram desconsiderados na análise dos dados, para evitar possíveis influências do processo de aceleração e desaceleração da marcha.

\section{Análise dos dados}

Dez ciclos de marcha foram obtidos em velocidade de preferência e utilizados para a análise dos dados. A determinação do tempo de passo, do tempo de passada, do tempo de apoio e do tempo de balanço foi realizada por meio do sinal dos sensores de pressão, com base na voltagem do sinal dos sensores de pressão $(5 \mathrm{mV}$ ou $0 \mathrm{mV})$. Os valores de variabilidade das respectivas variáveis foram calculados a partir do desvio-padrão.

A análise discriminativa foi realizada por meio do software PASW statistics $18.0^{\circledR}$ (SPSS), de modo a determinar um modelo preditor de alterações na marcha características da DP utilizando as variáveis tempo de passo, tempo de passada, tempo de apoio, tempo de balanço, variabilidade do tempo de passo, variabilidade do tempo de passada, variabilidade do tempo de apoio e variabilidade do tempo de balanço.

Calculou-se ainda a especificidade e sensibilidade de cada variável analisada e a curva receiver operating characteristic (ROC). A curva ROC está representada na Figura 2 e é obtida pela representação da sensibilidadexespecificidade. Altos valores de sensibilidade e especificidade, representados por uma maior área gráfica de sensibilidadexespecificidade, resultam em um modelo preditor mais significativo. $O$ ponto de corte da variável mais preditiva foi calculado por meio de análise estatística discriminativa por coeficientes, a qual aponta o valor limite capaz de discriminar idosos com e sem DP. O nível de significância estabelecido foi de $\mathrm{p}<0,05$ para todos os testes.

\section{RESULTADOS}

Os resultados mostraram que a variável com maior valor de sensibilidade e especificidade foi o tempo de balanço, o que pode classificá-lo como a variável estudada com maior capacidade preditiva da presença ou não da DP. O modelo preditor mais significativo é representado pela maior área gráfica (sensibilidadexespecificidade) entre as variáveis analisadas (Figura 2). O ponto de corte encontrado para a variável preditiva de tempo de balanço foi de 0,48 segundos. A Tabela 2 mostra os dados da área de curva ROC.

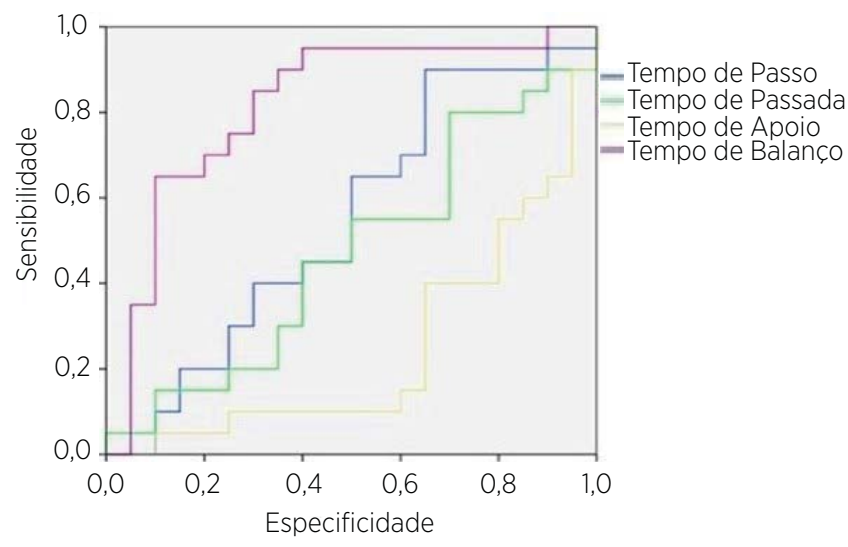

Figura 2. Curva Roc de especificidade e sensibilidade

Tabela 2. Dados de curva ROC

\begin{tabular}{lcccccc} 
& & & & \multicolumn{2}{c}{ IC 95\% } \\
\cline { 6 - 7 } \multicolumn{1}{c}{ Variáveis } & Área & EP & $p$ & LI & LS \\
\hline Tempo de passo & 0.545 & 0.093 & 0.626 & 0.362 & 0.728 \\
$\begin{array}{l}\text { Tempo de } \\
\text { passada }\end{array}$ & 0.470 & 0.093 & 0.745 & 0.288 & 0.652 \\
$\begin{array}{l}\text { Tempo de apoio } \\
\text { Tempo de }\end{array}$ & 0.245 & 0.078 & 0.006 & 0.092 & 0.398 \\
balanço & 0.818 & 0.072 & 0.001 & 0.677 & 0.958
\end{tabular}

EP: erro padrão; IC: intervalo de confiança; LI: limite inferior; LS: limite superior.

\section{DISCUSSÃO}

O objetivo deste estudo foi identificar, entre as variáveis cinemáticas temporais da marcha, aquelas capazes de discriminar idosos com e sem DP. Esta identificação é relevante, pois hoje o diagnóstico da DP é comumente feito de forma tardia, quando o indivíduo já apresenta comprometimentos motores identificáveis por uma avaliação ${ }^{17}$. Com a identificação precoce da DP, é possível programar precocemente a intervenção terapêutica, o que pode ajudar a prevenir ou minimizar complicações decorrentes da doença $\mathrm{a}^{18,19}$. 
Os resultados mostraram que o tempo de balanço é uma variável de alta sensibilidade e especificidade e, portanto, capaz de discriminar idosos com e sem DP. Idosos com DP apresentam um tempo de balanço menor que idosos sem DP, cujo ponto de corte encontrado foi de 0,48 segundos.

No estudo de Pistacchi et al. ${ }^{18}$ foram quantificados e identificados parâmetros espaço-temporais e cinemáticos da marcha em indivíduos saudáveis e com DP. A fase de balanço e o tempo de balanço diferiram consideravelmente $(\mathrm{p}<0,05)$, enquanto a fase de apoio não foi estatisticamente significativa em pacientes em comparação com indivíduos saudáveis. $\mathrm{O}$ tempo de balanço representa indiretamente a estabilidade e o equilíbrio funcional, haja vista que ele mostra o quanto o indivíduo é capaz de permanecer com apoio unipodal durante um ciclo de marcha. Portanto, quanto maior o tempo que o indivíduo necessita para se manter em base de apoio estável, ou bipodal, menor a sua capacidade de se manter equilibrado durante a atividade desempenhada ${ }^{19-21}$.

A instabilidade na marcha mostra-se como um sinal importante na DP, pois a maior parte dos pacientes não têm uma interação adequada dos sistemas que influenciam no equilíbrio dinâmico, como é o caso da marcha.

O déficit de equilíbrio na DP causa o deslocamento do centro de massa para frente, o que torna difícil a realização de movimentos compensatórios como forma de readquirir o equilíbrio ${ }^{22,23}$. Contudo, a estabilidade da marcha garante a capacidade de manter a locomoção funcional, apesar da presença de perturbações externas ou de erros de controle interno. A maior dificuldade de adaptação da marcha na população com DP é um risco considerável, principalmente no que se refere a quedas e consequências graves ${ }^{24}$.

Segundo a escala de H\&Y, os distúrbios do equilíbrio ocorrem apenas no terceiro estágio da DP. Entretanto, essa escala, amplamente utilizada na prática clínica, não foi desenvolvida para identificar alterações cinemáticas da marcha e, por isso, não é sensível às mudanças no tempo de balanço ${ }^{16}$. A esse respeito, em revisão feita por Kamieniarz et $a 1 .{ }^{25}$, foi identificado que a instabilidade postural pode aparecer já nos estágios iniciais da doença, mesmo antes do início dos sintomas clínicos, o que corrobora com os resultados aqui apresentados.

Faz-se importante esclarecer, contudo, que este estudo apresenta algumas limitações a serem consideradas na interpretação dos resultados. A análise cinemática foi realizada em ambiente laboratorial e tendo como referência o membro inferior dominante; entretanto, sabe-se que nas fases iniciais da DP as manifestações motoras não são simétricas bilateralmente. Em adição, todos os participantes eram fisicamente ativos por estarem inseridos em projetos de extensão; no entanto, esta não é a realidade da maioria dos idosos com ou sem DP.

\section{CONCLUSÃO}

A análise cinemática da marcha permite discriminar um grupo de indivíduos com DP de um grupo de indivíduos saudáveis, com alta sensibilidade e especificidade, mais especificamente no tempo de balanço, que é menor no grupo acometido pela doença (corte de 0,48segundos). A identificação de características anormais na marcha, principalmente no que se refere aos parâmetros cinemáticos relacionados com o equilíbrio dinâmico, como o tempo de balanço reduzido já nas fases iniciais da DP, pode ajudar a prever a evolução.

\section{REFERÊNCIAS}

1. O'Sullivan SB, Schimitz J. Fisioterapia: avaliação e tratamento. 3a ed. São Paulo: Manole; 1993.

2. Eygelshoven S, van den Hout A, Tucha L, Fuermaier ABM, Bangma DF, Thome J, et al. Are non-demented patients with Parkinson's disease able to decide about their own treatment? Park Relat Disord. 2017;38(1):48-53. doi: 10.1016/j. parkreldis.2017.02.02

3. Braak H, Del Tredici K, Rüb U, De Vos RAI, Jansen Steur ENH, Braak E. Staging of brain pathology related to sporadic Parkinson's disease. Neurobiol Aging. 2003;24(2):197-211. doi: 10.1016/s0197-4580(02)00065-9

4. Goedert M. Alpha-synuclein and neurodegenerative diseases. Nat Rev Neurosci. 2001;2(7):492-501. doi: 10.1038/35081564

5. Souza CFM, Almeida HCP, Sousa JB, Costa PH, Silveira YSS, Bezerra JCL. A doença de parkinson e o processo de envelhecimento motor: Uma revisão de literatura. Rev Neurocienc. 2011;19(4):718-23. doi: 10.34024/rnc.2011.v19.8330

6. Adams WR. High-accuracy detection of early Parkinson's Disease using multiple characteristics of finger movement while typing. PLoS One. 2017;30;12(11):188-226. doi: 10.1371/ journal.pone.0188226

7. Schreglmann SR, Bhatia KP, Stamelou M. Advances in the Clinical Differential Diagnosis of Parkinson's Disease. Int Rev Neurobiol. 2017;32:79-127. doi: 10.1016/bs.irn.2017.01.007

8. Schrag A, Horsfall L, Walters K, Noyce A, Petersen I. Prediagnostic presentations of Parkinson's disease in primary care: A casecontrol study. Lancet Neurol. 2015;14(1):57-64. doi: 10.1016/ S1474-4422(14)70287-X

9. Wert DM, Brach J, Perera S, Van Swearingen JM. Gait Biomechanics, Spatial and Temporal Characteristics, and the 
Energy Cost of Walking in Older Adults With Impaired Mobility. Phys Ther. 2010;7(1):977-85. doi: 10.2522/ptj.20090316

10. Plotnik M, Giladi N, Dagan Y, Hausdorff JM. Postural instability and fall risk in Parkinson's disease: Impaired dual tasking, pacing, and bilateral coordination of gait during the "ON" medication state. Exp Brain Res. 2011;210(3):529-38. doi: 10.1007/ s00221-011-2551-0

11. Waters R. Gasto de energia. In: Perry J, editor. Análise da marcha: função normal e patológica. Thorofare: Slack; 2004. p. 443-89.

12. Fearnley JM, Lees AJ. Ageing and parkinson's disease: Substantia nigra regional selectivity. Brain. 1991;114(5): 2283-301. doi: 10.1093/ brain/114.5.2283

13. Pagan FL. Improving outcomes through early diagnosis of Parkinson's disease. Am J Manag Care. 2012; 18(7):176-182.

14. Wild LB, De Lima DB, Balardin JB, Rizzi L, Giacobbo BL, Oliveira $\mathrm{HB}$, et al. Characterization of cognitive and motor performance during dual-tasking in healthy older adults and patients with Parkinson's disease. J Neurol. 2013;260(2):580-9. doi: 10.1007/ s00415-012-6683-3

15. Monteiro EP, Wild LB, Martinez FG, Pagnussat AS, PeyréTartaruga LA. Aspectos biomecânicos da locomoção de pessoas com doença de Parkinson: revisão narrativa. Rev Bras Cienc Esporte. 2017;39(4):450-457. doi: 10.1016/j.rbce.2016.07.003

16. Hoehn MM, Yahr M. Parkinsonism: onset, progression, and mortality. Neurology. 1967;17(5):427-42. doi: 10.1212/wnl.17.5.427

17. Hawkes CH, Del Tredici K, Braak H. A timeline for Parkinson's disease. Park Relat Disord. 2010;16(2):79-84. doi: 10.1016/j. parkreldis.2009.08.007
18. Pistacchi M, Gioulis M, Sanson F, Giovannini E, Filippi G, Rossetto F, et al. Gait analysis and clinical correlations in early Parkinson's disease. Funct Neurol. 2017;32(1):28-34. doi: 10.11138/fneur/2017.32.1.028

19. Frazzitta G, Balbi P, Maestri R, Bertotti G, Boveri N, Pezzoli G. The beneficial role of intensive exercise on Parkinson disease progression. Am J Phys Med Rehabil. 2013;92(6):523-32. doi: 10.1097/PHM.0b013e31828cd254

20. Kleiner A, Galli M, Gaglione M, Hildebrand D, Sale P, Albertini G, et al. The Parkinsonian Gait Spatiotemporal Parameters Quantified by a Single Inertial Sensor before and after Automated Mechanical Peripheral Stimulation Treatment. Parkinsons Dis. 2015;3(9):5-12. doi: 10.1155/2015/390512

21. Hollman JH, McDade EM, Petersen RC. Normative spatiotemporal gait parameters inolder adults. Gait and Posture. 2011; 34(1):111-8. doi: 10.1016/j.gaitpost.2011.03.024

22. Rodriguez KL, Roemmich RT, Cam B, Fregly BJ, Hass CJ. Persons with Parkinson's disease exhibit decreased neuromuscular complexity during gait. Clin Neurophysiol. 2013;124(7):1390-7. doi: 10.1016/j.clinph.2013.02.006

23. Warlop T, Detrembleur C, Bollens B, Stoquart G, Crevecoeur F, Jeanjean A, et al. Temporal organization of stride duration variability as a marker of gait instability in Parkinson's disease. J Rehabil Med. 2016;11;48(10):865-71. doi: 10.2340/16501977-2158

24. Słomka K, Juras G, Sobota G, Bacik B. The reliability of a rambling-trembling analysis of center of pressure measures. Gait Posture. 2013;37(2):210-3. doi: 10.1016/j.gaitpost.2012.07.005

25. Kamieniarz A, Michalska J, Brachaman A, Pawłowski M, Słomka KJ, Juras G. A posturographic procedure assessing balance disorders in Parkinson's disease: a systematic review. Clin Interv Aging. 2018;12(13):2301-16. doi: 10.2147/CIA.S180894 